\title{
Gold Nanoflares with Computing Function as Smart Diagnostic Automata for Multi-miRNA Patterns in Living Cells
}

Lan Liu, Na Li, Zhi-Mei Huang, Li-Juan Tang*, Zhan-Ming Ying, Jian-Hui Jiang*

State Key Laboratory of Chemo/ Bio-Sensing and Chemometrics, College of Chemistry and Chemical Engineering, Hunan University, Changsha 410082, P. R. China

*Corresponding Author: Fax: +86-731-88821916; E-mail: jianhuijiang@hnu.edu.cn, tanglijuang@hnu.edu.cn

\section{Table of Contents}

S-2. EXPERIMENTAL SECTION. Chemicals and materials, instruments and methods

S-5. Table S1. The DNA sequences

S-6. Figure S1. TEM image

S-7. Figure S2. UV-vis spectra analysis

S-8. Figure S3. Stability analysis

S-9. Figure S4. Fluorescence responses of the OR diagnostic automaton

S-10. Figure S5. Fluorescence responses of the AND diagnostic automaton

S-11. Figure S6. Quantitative analysis of the OR automaton with dual-miRNA targets

S-12. Figure S7. Quantitative analysis of the AND automaton with dual-miRNA targets

S-13. Figure S8. qRT-PCR analysis of related miRNAs in different cell lines

S-14. Figure S9. Relative miRNAs expression levels in different cell lines

S-15. Figure S10. Cytotoxicity assay

S-16. Figure S11. qRT-PCR analysis of related miRNAs in the regulated HeLa cells

S-17. Figure S12. Relative miRNAs expression levels in the regulated HeLa cells

S-18. Figure S13. Confocal images of diagnostic automata in the regulated HeLa cells. 


\section{EXPERIMENTAL SECTION}

Chemicals and Materials. All oligonucleotides were synthesized by Shanghai Sangon Biological Engineering Technology \& Services Co., Ltd, and purified by HPLC. Their sequences are listed in Table S1. The buffer for in vitro experiments was PBS (10 mM Phosphate buffer, $137 \mathrm{mM} \mathrm{NaCl}, 2.7$ $\mathrm{mM} \mathrm{KCl}, \mathrm{pH}$ 7.4). miR122 mimic and miR21 inhibitor were purchased from RiboBio Co. (Guangzhou, China). All reagents were used as received without further purification. All solutions were prepared using ultrapure water, obtained through a Millipore Milli-Q water purification system (Billerica, MA, USA) with an electric resistance $>18.2 \mathrm{M} \Omega$. Huh 7, HeLa and HEK 293T cells were obtained from the cell bank of Central Laboratory at Xiangya Hospital (Changsha, China). Cell culture media was obtained from Thermo Scientific HyClone (MA, USA).

Instruments. The transmission electron microscopic (TEM) images were obtained on a JEM-2100 transmission electron microscope (JEOL Ltd., Japan). The steady-state fluorescence measurements were carried out at room temperature in a quartz cuvette on an FL-7000 spectrometer (Hitachi, Japan). For the real-time fluorescence intensity study, Tecan Finite M1000 (Tecan, Switzerland) was used. Agarose gel was visualized via a Tanon 4200SF gel imaging system (Tanon Science \& Technology Co., Ltd., China). Fluorescence images of OR/AND diagnostic automaton in 98 wells were collected by an IVIS Lumina II in vivo imaging system (Caliper LifeSicence, USA). All cell fluorescence images were acquired using an oil dipping objective (60×) on Nikon TI-E+A1 SI confocal laser scanning microscope (Japan). The cell viability assays were operated on Thermo Scientific Multiscan Microplate Reader (Thermo Fisher, USA).

Synthesis of Gold Nanoparticles (AuNPs) and diagnostic gold nanoflares. The citrate-stabilized gold nanoparticles (AuNPs, $13 \mathrm{~nm}$ ) were prepared according to previous report method. The automata were synthesized as follows: $25 \mu \mathrm{M}$ thiolated strand $\mathbf{S}$ and the corresponding $\mathbf{F}$ strand (TMARA-Fo for the OR diagnostic automaton, TAMRA-F $F_{A}$ for the AND diagnostic automaton) were incubated in $1 \mathrm{x}$ PBS buffer at $37^{\circ} \mathrm{C}$ for $2 \mathrm{~h}$, therefore, formed the S-F duplex. After reduced by Tris (2-carboxyethyl) phosphine hydrochloride (TCEP· $\mathrm{HCl}$ ), the $60 \mu \mathrm{L}$ S-F duplex were slowly added to $450 \mu \mathrm{L}$ of $10 \mathrm{nM}$ AuNPs. The mixture was incubated at room temperature and shaken overnight. $27 \mu \mathrm{L}$ phosphate buffer (200 mM, pH 7.4) and $1 \mu \mathrm{L}$ SDS (10\%) were then added to the mixtures. After $1 \mathrm{~h}, 10 \mathrm{mM}$ phosphate with $2 \mathrm{M} \mathrm{NaCl}$ were gradually added to the solution during a period of $12 \mathrm{~h}$, the obtained final concentration of $\mathrm{NaCl}$ is $0.3 \mathrm{M}$. The mixture was shaken for another $12 \mathrm{~h}$. Finally, the mixture was centrifuged (1,5000 rpm, $30 \mathrm{~min})$ for three times and resuspended in PBS buffer. The concentration of synthesized automata was determined by measuring their extinction at $523 \mathrm{~nm}\left(\varepsilon=2.7 \times 10^{8} \mathrm{~L} \mathrm{~mol}^{-1}\right.$ $\left.\mathrm{cm}^{-1}\right)$.

Gel electrophoresis analysis. Agarose gel electrophoresis was used to evaluate the performance of the basic DNA reactions. $10 \mu \mathrm{L}$ DNA samples were mixed with $2 \mu \mathrm{L} 6 \mathrm{x}$ loading buffer and then loaded 
into $4.5 \%$ agarose gel which was stained by $0.5 \mu \mathrm{g} / \mathrm{mL}$ GoldView and $0.5 \mu \mathrm{g} / \mathrm{mL}$ ethidium bromide. The duplex S-F was formed at $5 \mu \mathrm{M}$ by incubating the solution at $37^{\circ} \mathrm{C}$ for $2 \mathrm{~h}$, and then used for the following reactions. The concentration of each strand in lane 1-4 in Figure 1 (miR122, miR21, S and F) was $2.5 \mu \mathrm{M}$. The final concentration of each strand in lane 5-11 was $1 \mu \mathrm{M}$.

Fluorescence Measurements. The concentration of the OR/AND diagnostic automaton used in the in vitro fluorescence measurements was $1 \mathrm{nM}$. The concentration of miRNAs used in Figure 2, S4 and S5 was $200 \mathrm{nM}$. Two miRNA targets of same concentration were added at the same time in Figure S6, S7. The fluorescence emission spectra were collected from $550 \mathrm{~nm}$ to $650 \mathrm{~nm}$ at room temperature with a $535 \mathrm{~nm}$ excitation wavelength. The kinetic study was performed at $37^{\circ} \mathrm{C}$ by excitation with a $535 \mathrm{~nm}$ excitation. The time interval was set as $30 \mathrm{~s}$.

Cell culture. Huh 7 cells (human hepatoma cell line), HeLa cells (human cervical carcinoma cell lines), HEK 293T cells (human renal epithelial cells) were cultured in RPMI 1640 medium (Thermo Scientific Hyclone) supplemented with 10\% fetal bovine serum (Invitrogen). The cells were cultured at $37^{\circ} \mathrm{C}$ in a humidified atmosphere containing $5 \% \mathrm{CO}_{2}$.

Confocal fluorescence imaging. The cells were plated on sterilized glass coverslips in $35 \mathrm{~mm}$ plates with $14 \mathrm{~mm}$ well and grown to 50-70\% confluency in the corresponding medium for $24 \mathrm{~h}$. Next, the cells were incubated with $1 \mathrm{nM}$ OR/AND diagnostic automaton at $37{ }^{\circ} \mathrm{C}$ in $5 \% \mathrm{CO}_{2}$ for $6 \mathrm{~h}$, and then the cells were washed three times with PBS ( $\mathrm{pH}=7.4)$ before imaging. In order to modulate the expression of miR21 and miR122 in the HeLa cells, synthetic miR122 mimic or miR21 inhibitor of a given concentration was transfected by lipofectamine 3000 for $24 \mathrm{~h}$. Then, the cells were incubated with $1 \mathrm{nM}$ OR/AND diagnostic automaton for $6 \mathrm{~h}$. After washed three times with PBS, the cells were imaged on confocal laser scanning microscope. The data was then analyzed by NIS-Viewer. Normalized imaging data were collected and processed by image Image J. The average and standard deviation were obtained by 3 parallel cell fluorescent imaging experiments.

Quantitative reverse transcription-PCR (qRT-PCR) analysis. Total cellular RNA was extracted using the SanPrep Column microRNA Extraction Kit (Sangon, China) according to its manual. The cDNA samples were prepared with a Revert Aid Premium Reverse Transcriptase kit (Thermo Scientific) according to the indicated protocol. The cDNA samples were diluted 10 times before use. qPCR analysis of cDNA was performed with SybrGreen Fast qPCR Master Mix (ABI, USA) on an ABI Stepone Plus qPCR instrument. A total volume of $20 \mu \mathrm{L}$ reaction solution contains $2 \mu \mathrm{L}$ cDNA sample, $10 \mu \mathrm{L}$ SybrGreen qPCR Master Mix, $0.4 \mu \mathrm{L}$ forward transcription primer $(10 \mu \mathrm{M}), 0.4 \mu \mathrm{L}$ reverse transcription primer $(10 \mu \mathrm{M})$ and $7.2 \mu \mathrm{L}$ nuclease-free waster. The PCR conditions were as follows: an initial $95{ }^{\circ} \mathrm{C}$ for $3 \mathrm{~min}$ followed by 45 cycles of $95^{\circ} \mathrm{C}$ for $3 \mathrm{~s}, 60{ }^{\circ} \mathrm{C}$ for $30 \mathrm{~s}$. The endogenous gene U6 was used as control for quantifying the relative level of miRNAs. The primers used were: U6-F, 5'-CTC GCT TCG GCA GCA CA-3'; U6-R, 5’- AAC GCT TCA CGA ATT TGC GT-3’; miR21-RT, 5’-CTC 
AAC TGG TGT CGT GGA GTC GGC AAT TCA GTT GAG TCA ACA TCA-3'; miR21-F, 5'-ACA CTC CAG CTG GGT AGC TTA TCA GAC TG -3'; miR122-RT, 5'-CTC AAC TGG TGT CGT GGA GTC GGC AAT TCA GTT GAG CAA ACA CC-3'; miR122-F, 5'-ACA CTC CAG CTG GGT GGA GTG TGA CAA T -3; All R, 5'-TGG TGT CGT GGA GTC G-3'. We evaluated all the data with respect to the microRNA expression by normalizing to the expression of U6 and using the $2^{-\triangle \triangle} \mathrm{Ct}$ method.

Cytotoxicity Assay. The cell viability was determined using a CellTiter 96® AQueous One Solution Cell Proliferation Assay kit. In the assay, cells were seeded in 96-well plates at $4 \times 10^{3}$ cells per well and incubated overnight before treatments. Growth medium (50 $\mu \mathrm{L}$ per well) containing $5 \mathrm{nM}$ OR or AND diagnostic automaton were added in cell-plated wells. After 12, 18 or 24 hours, $20 \mu \mathrm{L}$ CellTiter Reagent diluted with $50 \mu \mathrm{L}$ of growth medium was added per well and incubated with the cells at $37^{\circ} \mathrm{C}$ for $1 \mathrm{~h}$ and then measured. 
Table S1. Sequences of DNA probes. ${ }^{\mathrm{a}, \mathrm{b}}$

\begin{tabular}{|c|c|}
\hline Name & Sequence (5’ -3’ ) \\
\hline \multirow{3}{*}{ miR21 } & TAGCTTATCAGACTGATGTTGA \\
\hline & TAGCTTATCAGACT - GATGTTGA (OR, denoted by $1 a-1 b)$ \\
\hline & TAGCTTA - TCAGACTGATGTTGA (AND, denoted by $1 m-1 n$ ) \\
\hline \multirow{3}{*}{ miR122 } & TGGAGTGTGACAATGGTGTTTG \\
\hline & TGGAGTGT - GACAATGGTGTTTG (OR, denoted by 2a-2b) \\
\hline & TG, GAG - TGTGACAATGGTGTTTG (AND, denoted by $2 m_{1}, 2 m_{2}-2 n$ ) \\
\hline miR122-m1 & TGIAGTGTGACAATGGTGTTTG \\
\hline miR122-m3 & TGIAㅌTGTGAAAATGGTGTTTG \\
\hline $\operatorname{miR} 126$ & TCGTACCGTGAGTAATAATGCG \\
\hline \multirow{3}{*}{$\mathbf{S}$} & $\begin{array}{l}\text { CAAACACCATTGTCACACTCCATCAACATCAGTCTGATAAGCTATTTTTTTTT } \\
\mathrm{T}\end{array}$ \\
\hline & $\begin{array}{l}\text { CAAACACCATTGTC - ACACTCCA - TCAACATC - AGTCTGATAAGCTA - } \\
\left.\text { TTTTTTTTTT (OR, denoted by } 2 b^{*}-2 a^{*}-1 b^{*}-1 a^{*}-T_{10}\right)\end{array}$ \\
\hline & $\begin{array}{l}\text { CAAACACCATTGTCA - CA, CTC - CATCAACATCAGTCTGA - TAAGCTA - } \\
\text { TTTTTTTTTT (AND, denoted by } 2 n^{*}-2 m_{1}, 2 m_{2} *_{-}-1 n^{*}-1 m^{*} T_{10} \text { ) }\end{array}$ \\
\hline SH-S & $\begin{array}{l}\text { CAAACACCATTGTCACACTCCATCAACATCAGTCTGATAAGCTATTTTTTTTT } \\
\mathrm{T}-\mathrm{SH}\end{array}$ \\
\hline Fo & $\begin{array}{l}\text { ACTAGTGC - GATGTTGA - TGGAGTGT - GCACTAGT (OR, denoted by } \\
\left.x-1 b-2 a-x^{*}\right)\end{array}$ \\
\hline TAMRA-Fo & 6-TMARA-ACTAGTGCGATGTTGATGGAGTGTGCACTAGT \\
\hline $\mathbf{F}_{\mathbf{A}}$ & $\begin{array}{l}\text { TCAGACTGATGTTGA - TG - TGTGACAATGGTGTTTG (AND, denoted by } \\
\left.1 n-2 m_{1}-2 n\right)\end{array}$ \\
\hline TAMRA-FA & 6-TMARA-TCAGACTGATGTTGA TGTGTGACAATGGTGTTTG \\
\hline
\end{tabular}

${ }^{a}$ The mismatched nucleotide is marked with underline.

${ }^{b}$ Note: in the detailed design of AND gate, toehold region " $2 m$ " for miR122 was divided into two segments (“ $2 m_{1}$ ” and “ $2 m_{2}$ ”, with length of $2 \mathrm{nt}, 3 \mathrm{nt}$, separately). In $\mathbf{S}$ strand, “ $2 m_{1}$ *” was protected by $\mathbf{F}_{\mathbf{A}}$, and the unpaired " $2 m_{2}$ " part created a small bulge in the $\mathbf{S}-\mathbf{F}_{\mathbf{A}}$ duplex. In the presence of miR21, $\mathbf{F}_{\mathbf{A}}$ was partially displaced owing to toehold mediate strand migration, " $2 m_{1}$ *” became unpaired because of the unstable hybridization. Thus the whole toehold region “ $2 m$ *” was exposed subsequently. This design ensures the efficiency of the TMSD reaction, as well as decreases the side reactions. 
Figure S1. TEM image of the AuNPs.

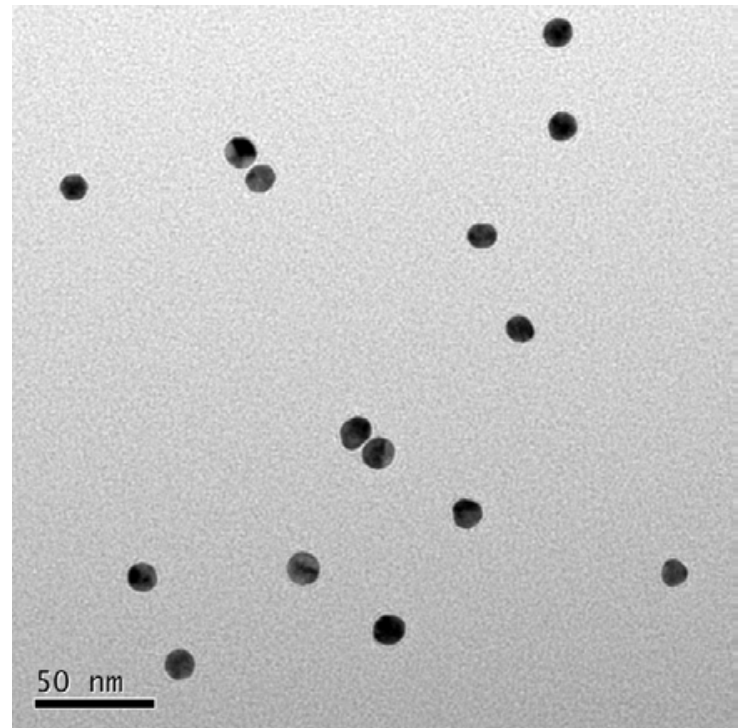


Figure S2. UV-vis spectra for bare AuNPs (black) and AND automaton(red) and OR automaton(blue).

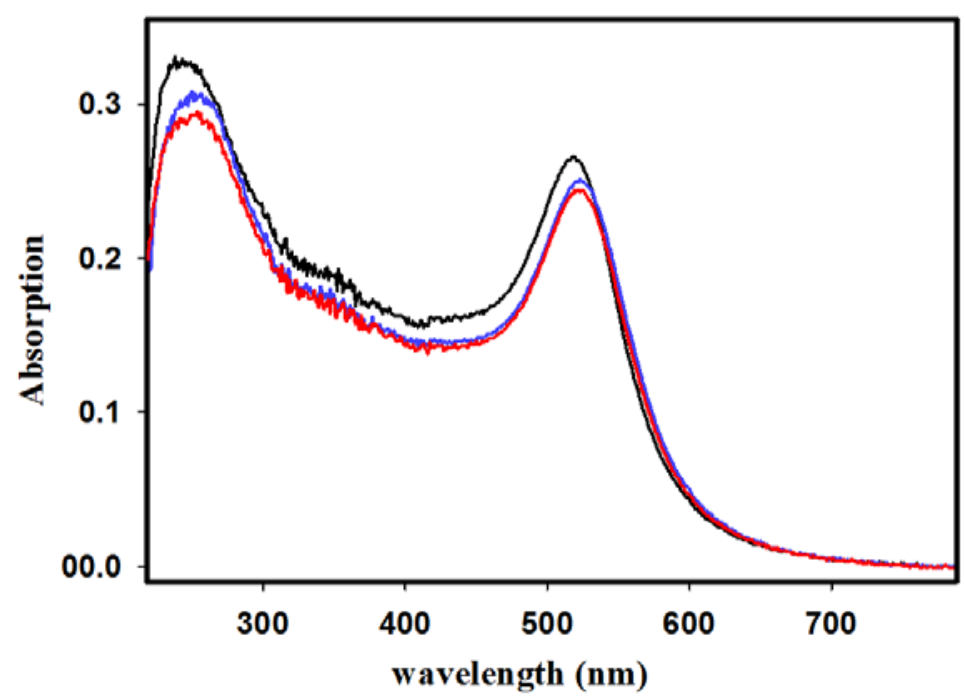


Figure S3. Stability analysis of the $\mathrm{OR}(\mathrm{A})$ and $\mathrm{AND}(\mathrm{B})$ automata. (1) Fluorescence intensity at different time point after being synthesized, (2) photograph of the automata after being synthesized, (3) after storage for 1 week at room temperature.

A1

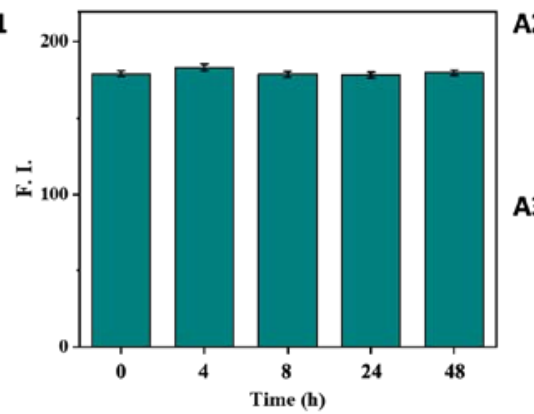

B1

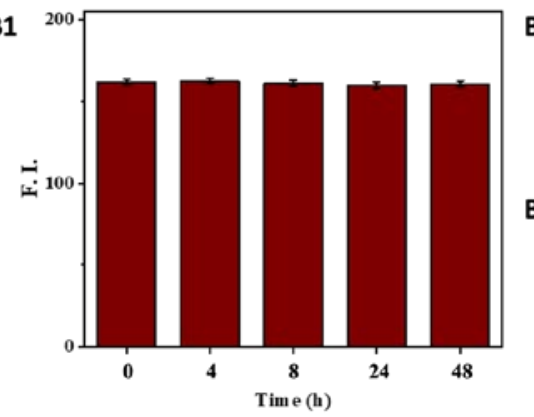

A2

B2

B3

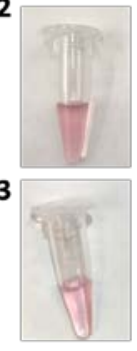


Figure S4. Fluorescence spectral responses obtained by incubating the OR diagnostic automaton (orange), with miR21 (blue), miR122 (green), miR122 and miR21 (red), miR122-m1(cyan), miR122-m3(purple), miR126(pink).

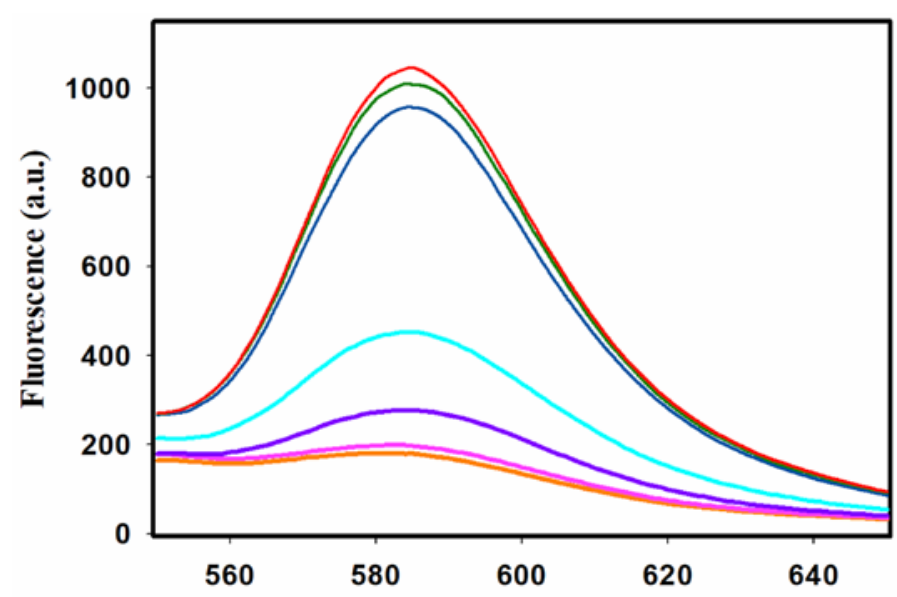


Figure S5. Fluorescence spectral responses obtained by incubating the AND diagnostic automaton (black), with miR21 (blue), miR122 (dark green), miR122 and miR21 (red), miR122-m1(orange), miR122-m3(light green), miR126(pink).

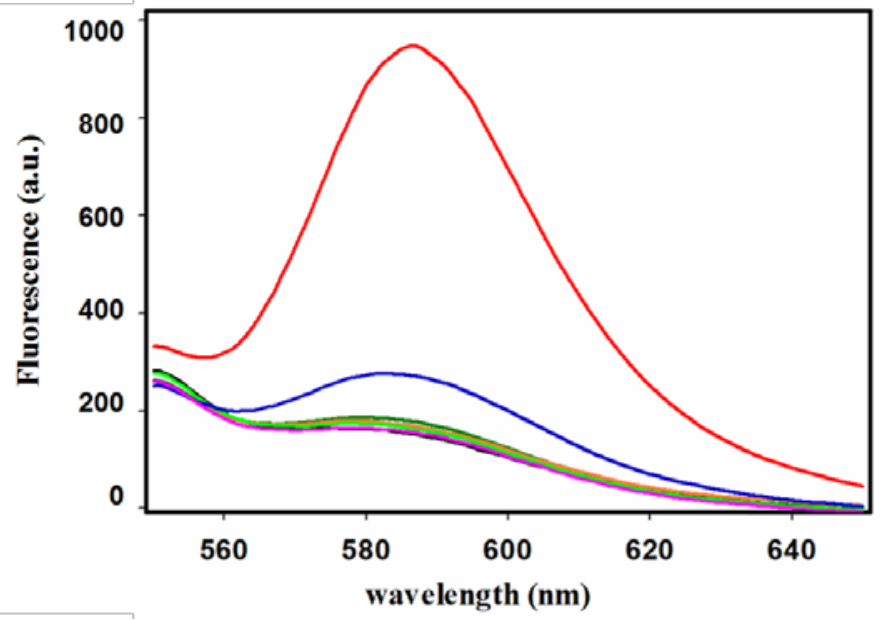


Figure S6. (A)Fluorescence spectral responses of the OR diagnostic automaton for targets of varying concentrations in a dual-miRNA mode with a 1:1 ratio. (B) Plot of fluorescence peak intensities versus the corresponding concentrations.
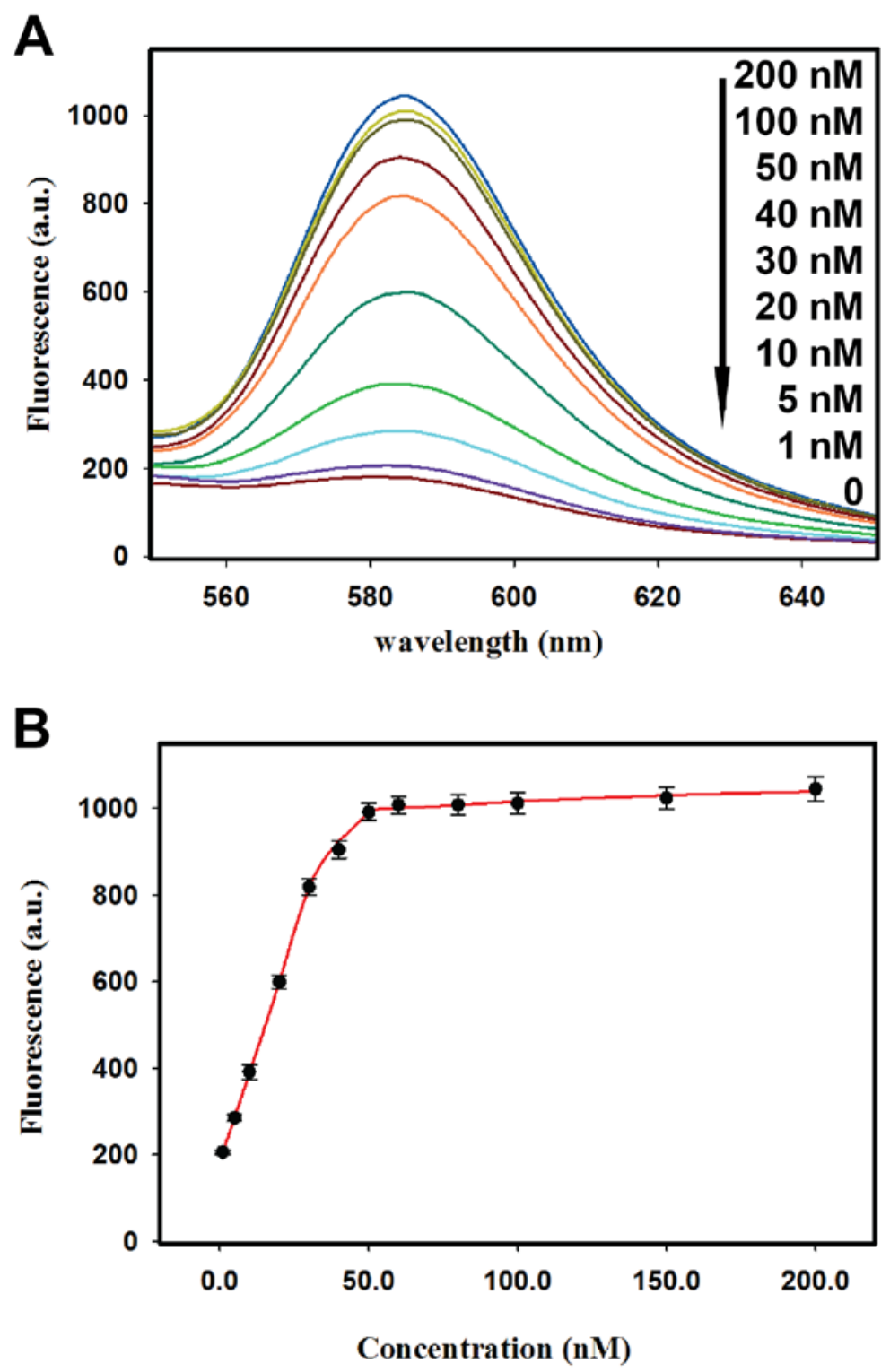
Figure S7. (A) Fluorescence spectral responses of the AND diagnostic automaton for targets of varying concentrations in a dual-miRNA mode with a 1:1 ratio. (B) Plot of fluorescence peak intensities versus the corresponding concentrations.
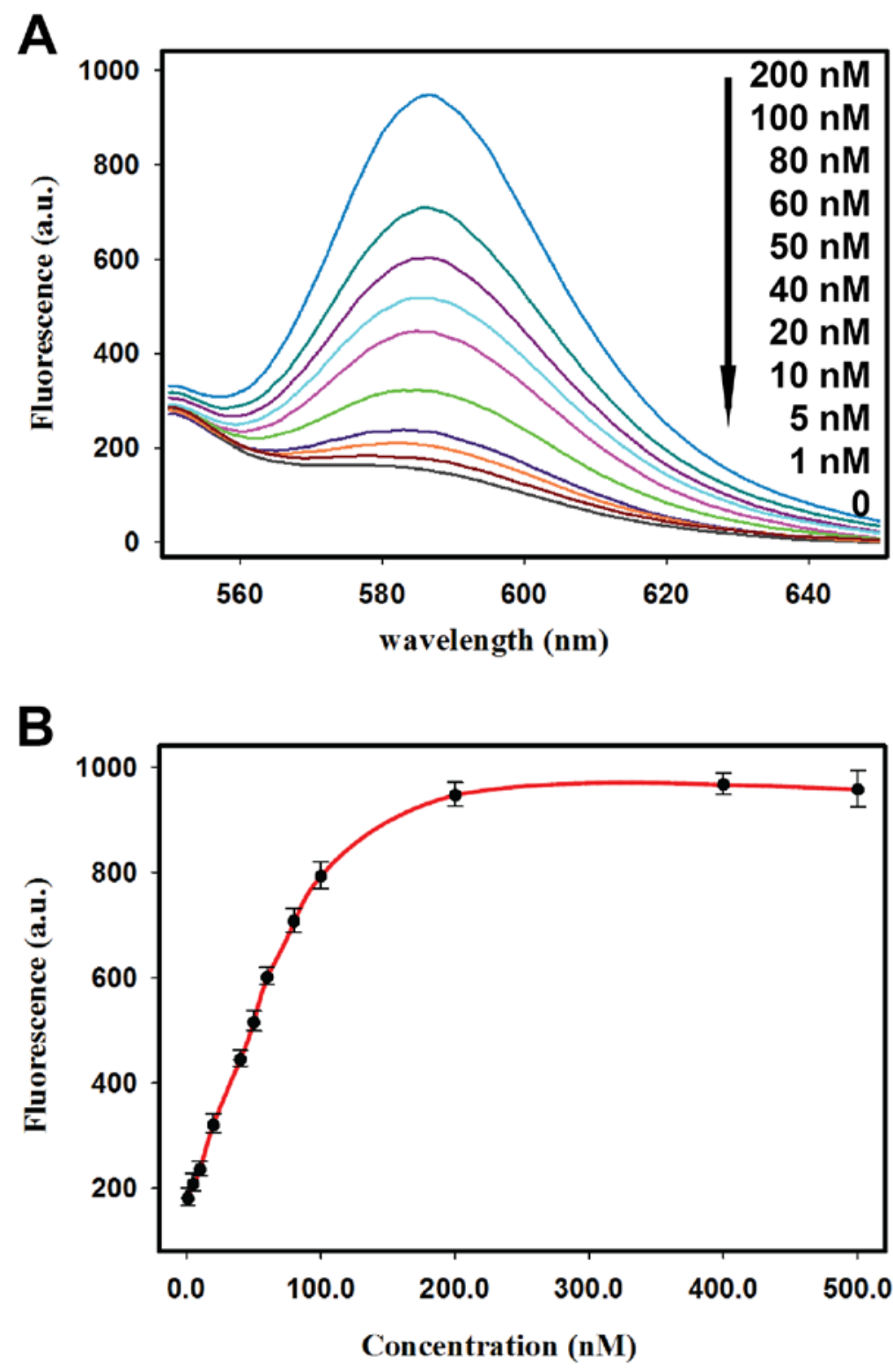
Figure S8. Real-time fluorescence curves in qRT-PCR analysis of (A) U6, (B) miR21and (C) miR122 in Huh 7 cells (red curves), Hela cells (orange curves) and HEK 293T cells (green curves). Three measurements have been done in each group.
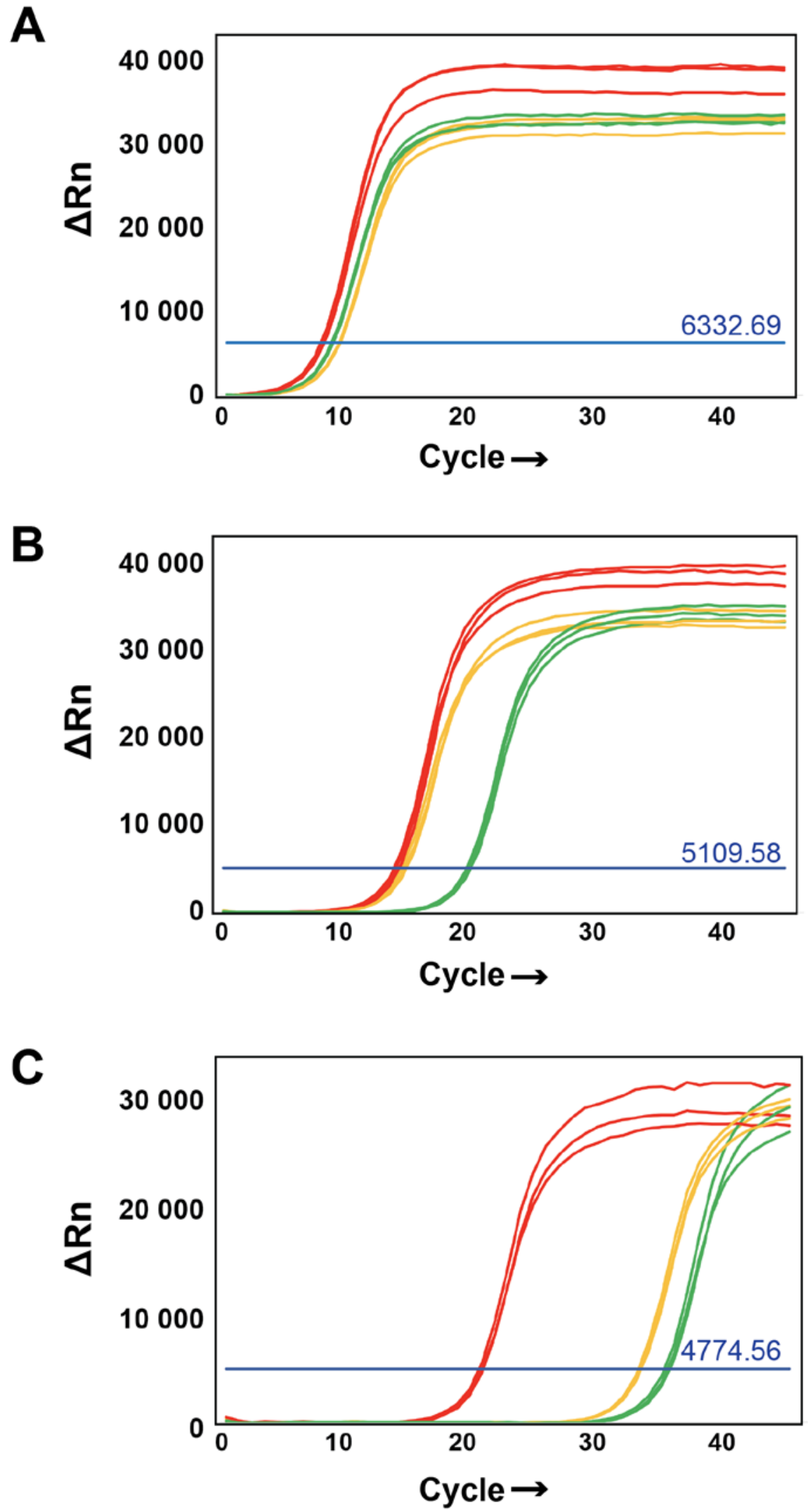
Figure S9. Relative expression levels for miR21 and miR122 in different cell lines according to the qRT-PCR results. The relative level of miR21 and miR122 were normalized to the expression level in Huh 7 cells.

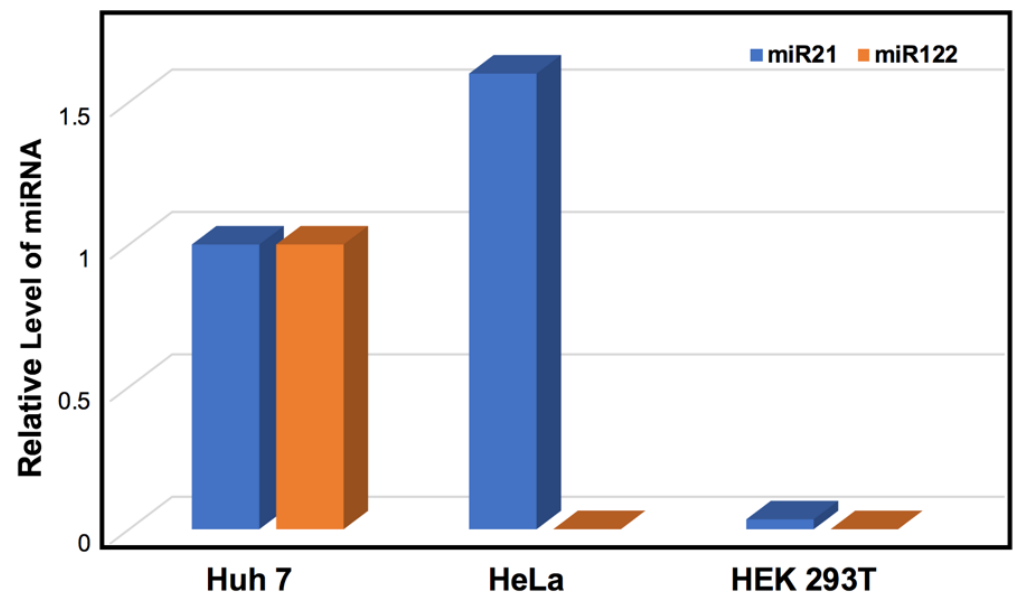


Figure S10. Cytotoxicity assay. HeLa cells incubated with $5 \mathrm{nM} \mathrm{OR/AND} \mathrm{diagnostic} \mathrm{automaton} \mathrm{for} \mathrm{12,}$ 18 or 24 hours, respectively. Error bars represent variations between three measurements. Three measurements have been done in each group.

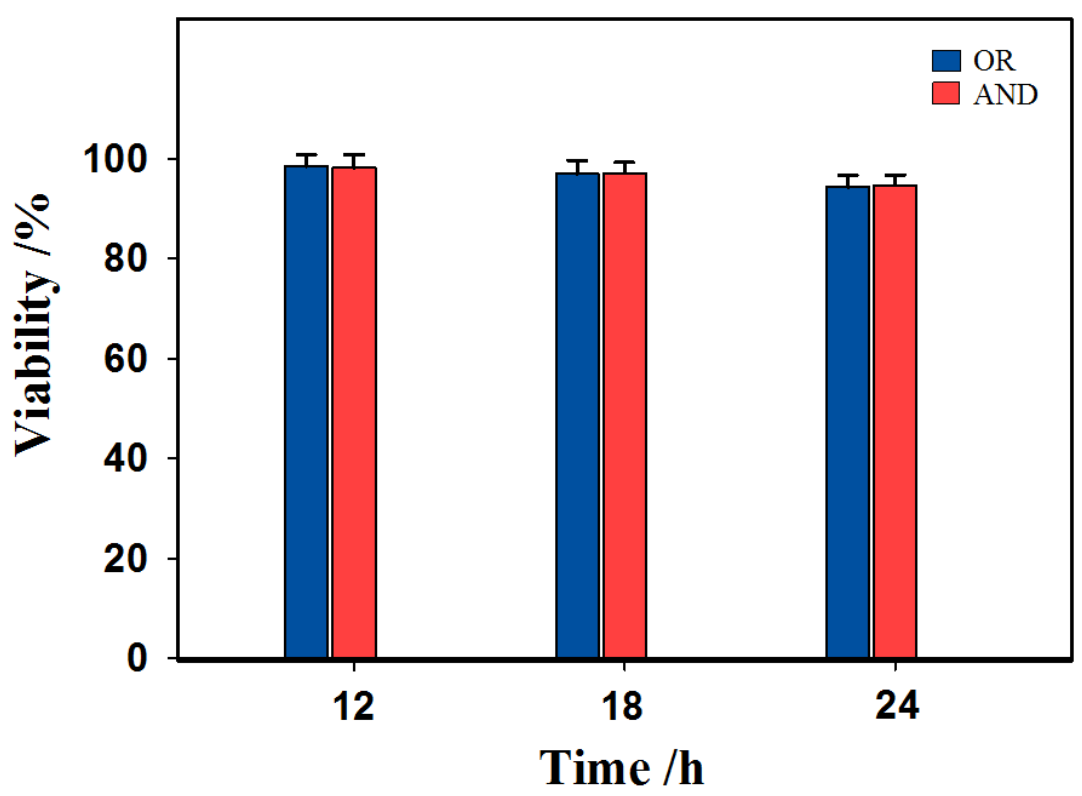


Figure S11. Real-time fluorescence curves in qRT-PCR analysis of (A) U6, (B) miR21and (C) miR122 in untreated HeLa cells (orange curves), cells treated with miR21 inhibitor (blue curves) and cells treated with miR122 mimic (purple curves).

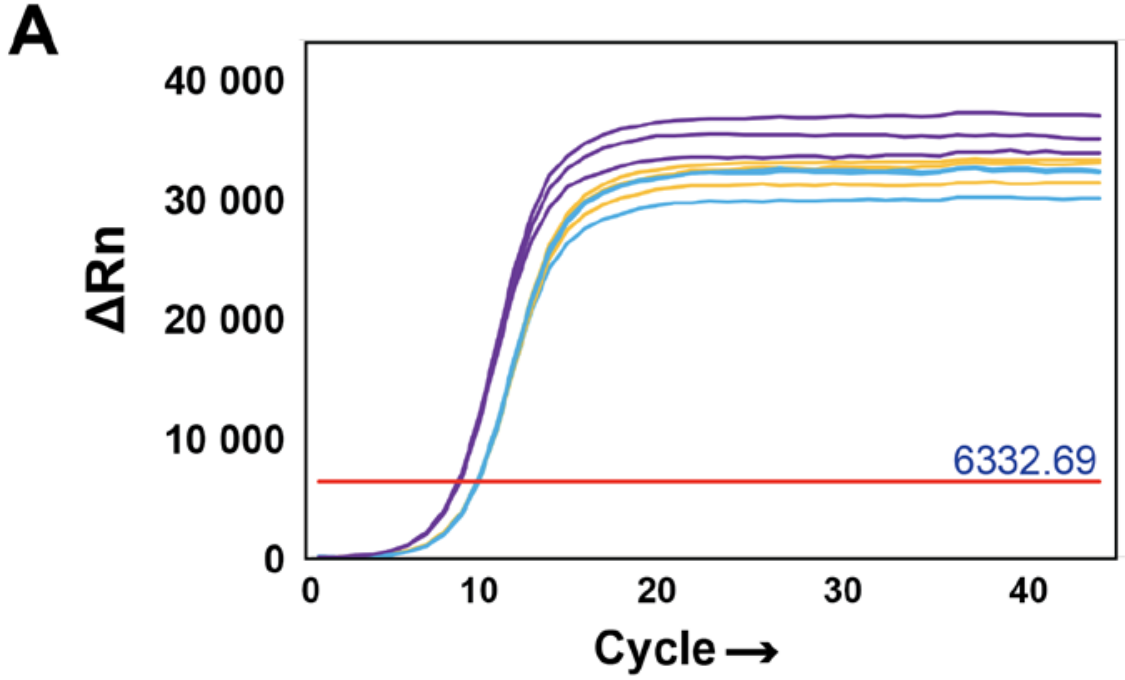

B

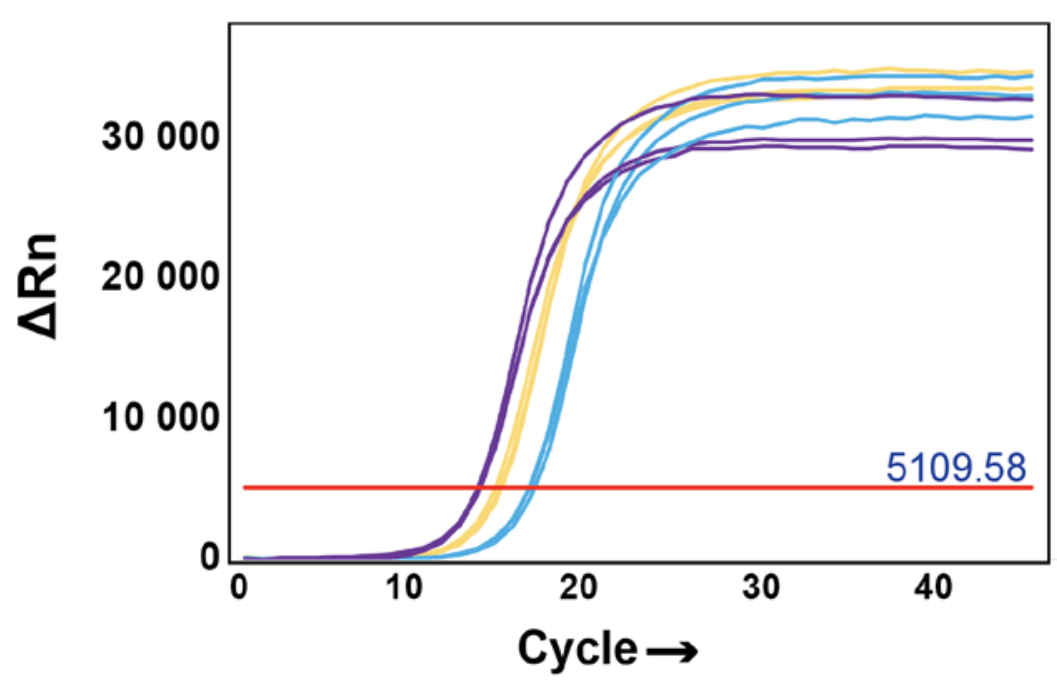

C

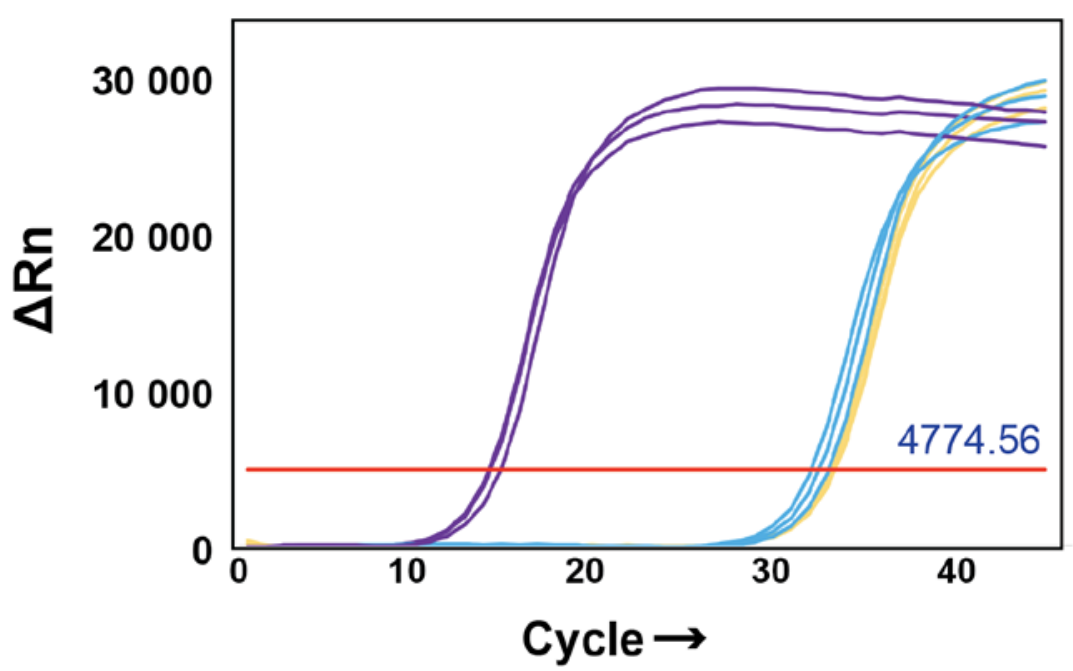


Figure S12. Relative expression levels of miR21 and miR122 in the untreated/treated HeLa cells according to the qRT-PCR results. The relative level of miR21 was normalized to the expression level in the untreated cells and the relative level of miR122 was normalized to the expression level in miR122 mimic treated ones.

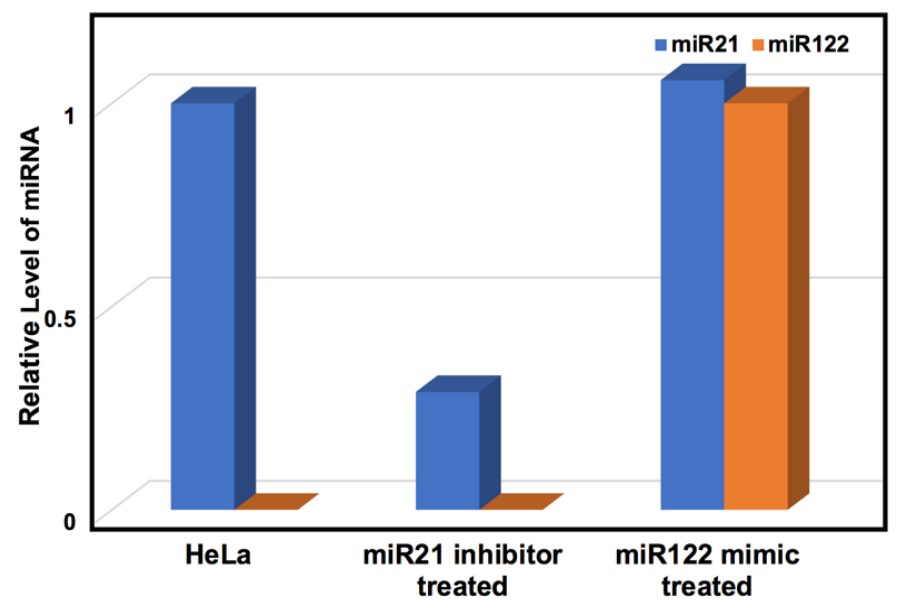


Figure S13. Confocal images of OR (1) and AND (2) diagnostic automata in different groups of HeLa cell lines: no treatment (A), miR122 mimic treated (B) and miR21 inhibitor treated (C) cells. Scale bar indicates $50 \mu \mathrm{m}$. (3) Histogram of the relative fluorescence intensity.

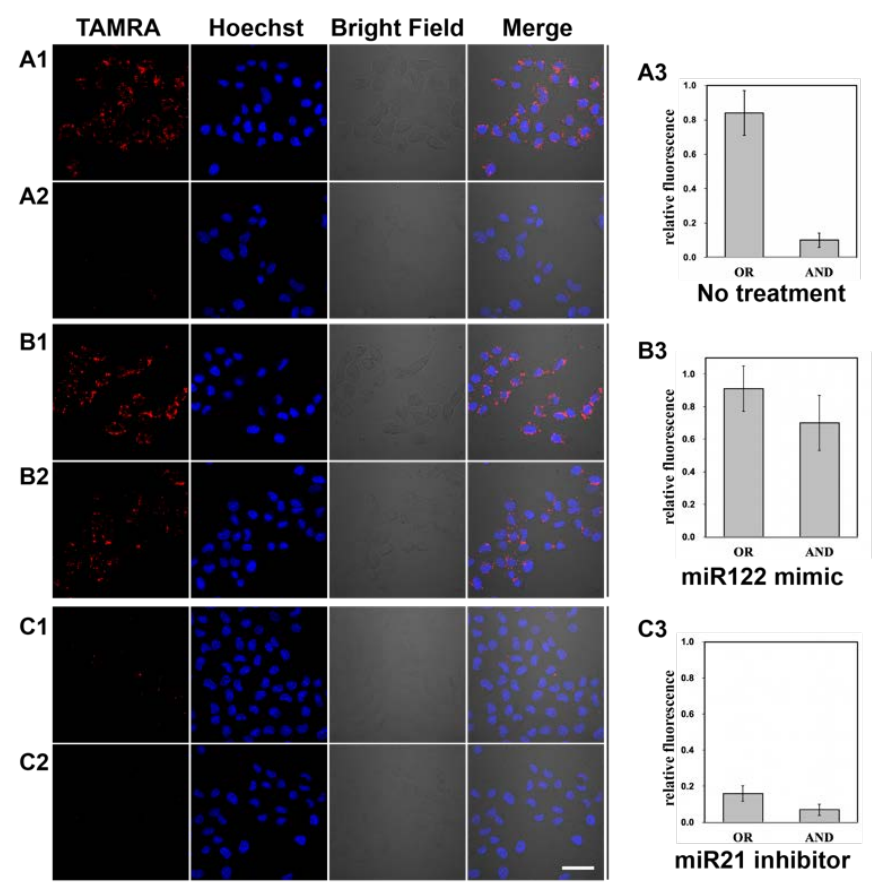

